\title{
BMJ Open Anxiety and depression among healthcare workers during the COVID-19 pandemic: a systematic umbrella review of the global evidence
}

\author{
Ritin Fernandez (D) , 1,2,3 Nqobile Sikhosana, ${ }^{2,3}$ Heidi Green, ${ }^{2,3}$ \\ Elizabeth J Halcomb (1D , ${ }^{1,2,4}$ Rebekkah Middleton, ${ }^{1,2,4}$ Ibrahim Alananzeh, ${ }^{1}$ \\ Stamatia Trakis, ${ }^{2}$ L Moxham ${ }^{1,2,3,4}$
}

To cite: Fernandez $\mathrm{R}$, Sikhosana N, Green $\mathrm{H}$, et al. Anxiety and depression among healthcare workers during the COVID-19 pandemic: a systematic umbrella review of the global evidence. BMJ Open 2021;11:e054528. doi:10.1136/ bmjopen-2021-054528

- Prepublication history for this paper is available online. To view these files, please visit the journal online (http://dx.doi org/10.1136/bmjopen-2021 054528).

Received 22 June 2021 Accepted 31 August 2021
Check for updates

(C) Author(s) (or their employer(s)) 2021. Re-use permitted under CC BY-NC. No commercial re-use. See rights and permissions. Published by BMJ.

For numbered affiliations see end of article.

Correspondence to Dr Ritin Fernandez; ritin@uow.edu.au

\section{ABSTRACT}

Objectives To summarise the evidence relating to the prevalence of anxiety and depression among healthcare workers (HCWs) during the COVID-19 pandemic. Design An umbrella review of systematic reviews was undertaken using the Joanna Briggs Institute (JBI) methods.

Data sources The Cochrane database of systematic reviews, JBI Evidence Synthesis, MEDLINE, Web of Science, PsycINFO, Embase and CINAHL were searched in March 2021 for reviews published in English.

Eligibility criteria Systematic reviews reporting the prevalence of anxiety and depression among HCWs during the COVID-19 pandemic. Two researchers screened each abstract and independently reviewed full text articles. Study quality was assessed using the JBI critical appraisal tool for systematic reviews, and the degree of overlap in primary studies was calculated.

Results Ten systematic reviews (100 unique studies), including 169157 HCWs from 35 countries were included. The prevalence of anxiety among all HCWs ranged from $22.2 \%(95 \% \mathrm{Cl} 21.3 \%$ to $23.1 \%)$ to $33.0 \%(95 \% \mathrm{Cl} 31.9 \%$ to $34.1 \%)$. The prevalence of anxiety among physicians $(n=5820)$ was reported to be between $17 \%$ and $19.8 \%$ and for nurses $(n=14938)$ between $22.8 \%$ and $27 \%$. The prevalence of depression among all HCWs ranged from $17.9 \%(95 \% \mathrm{Cl} 17.1 \%$ to $18.8 \%$ ) to $36 \%$ (95\% Cl $34.9 \%$ to $37.1 \%)$. The prevalence of depression among physicians $(n=643)$ and nurses $(n=8063)$ was reported to be $40.4 \%$ and $28 \%$, respectively.

Conclusions There is wide variation evident in the presence of anxiety and depression among HCWs. In particular, the prevalence of depression among physicians was high. Strategies to reduce the incidence of anxiety and depression are urgently required.

PROSPERO registration number CRD42021238960.

\section{INTRODUCTION}

COVID-19 was declared a pandemic by the WHO on 11 March 2020 and with, limited knowledge about the virus, its rapid spread and high mortality, people around the world became increasingly anxious. ${ }^{1}$ While
Strengths and limitations of this study

- This is an up to date comprehensive umbrella review on the prevalence of anxiety and depression among healthcare workers during the COVID-19 pandemic.

- The systematic literature search was conducted in seven major databases using database-specific filters.

- Methodological quality of the included reviews was assessed using a standardised measure.

- The degree of overlap in primary studies was assessed and calculated using the corrected covered area index method.

The primary studies were predominantly from China.

anxiety increased among the general population, at the frontline, healthcare workers (HCWs) experienced heightened emotional responses as they were more frequently exposed to the virus. ${ }^{2}$ Additionally, challenges in accessing appropriate supplies of personal protective equipment (PPE), job stress due to increased work demands and lack of effective treatment, working under rapidly changing COVID-19 protocols, risk of being infected and infecting their families, treating and comforting infected patients and decline in their immunity as a result of physical and mental exhaustion or underlying health conditions amplified anxiety among HCWs. ${ }^{3-6}$

Although the numbers are difficult to quantify, worldwide, as of May 2020 over 152888 HCWs have been reported to be infected with COVID-19 and more than 1413 COVID-19 associated deaths have been recorded. ${ }^{7}$ This risk of morbidity and mortality related to COVID-19 has consequences for mental health among HCWs. ${ }^{8-10}$ Previous epidemics such as Severe Acute Respiratory Syndrome (SARS) and Middle 
East Respiratory Syndrome (MERS) have been reported to have exacerbated or precipitated mental health problems among HCWs. ${ }^{11} 12$ Unlike these previous epidemics, the significant mortality rate among HCWs along with the high levels of media coverage and extensive global connectivity have substantially contributed to high levels of anxiety and fear about COVID-19. ${ }^{13}$

Data from previous epidemics reveal a high risk of anxiety which can lead to burnout and mental exhaustion culminating in staff leaving the profession. Previous research has also documented HCWs developing posttraumatic stress disorder and post-traumatic stress symptoms a few months after exposure to MERS and SARS. ${ }^{14}$

Numerous primary studies followed by systematic reviews have been conducted to identify the mental wellbeing of HCWs during the COVID-19 pandemic. An umbrella review of systematic reviews ${ }^{15}$ has been previously conducted, however, the methodological limitations of the review and the increase in systematic reviews involving global data necessitated a rigorously conducted umbrella review. Therefore, the objectives of this paper are to conduct an umbrella review of systematic reviews, to identify the prevalence of mental health disorders, to enhance the development of targeted interventions and to alleviate the emotional burden of HCWs.

\section{METHODS}

The Preferred Reporting Items for Systematic Reviews and Meta-Analyses ${ }^{16}$ was used to report this review. Additionally, this review was conducted in accordance with the Joanna Briggs Institute (JBI) umbrella review methodology. ${ }^{17}$ The review protocol was registered in the International Prospective Register of Systematic Reviews.

\section{Search strategy}

A three-phase search process was conducted in March 2021 to identify systematic reviews that reported on the prevalence of anxiety and depression among HCWs during COVID-19. First, initial keywords were identified, followed by analysis of the text words contained in the title and abstract, and of the index terms. Second, databasespecific search filters were constructed, and finally the reference list of all included reviews was searched. The databases searched were: Cochrane database of systematic reviews, JBI Evidence Synthesis, MEDLINE, Web of Science, PsycINFO, Embase and CINAHL. The federated search engine Epistemonikos, which specifically targets research syntheses, was also used. The key terms used were healthcare workers, health workers, health professionals, nurses, doctors, coronavirus, COVID-19, SARS-CoV-2, psychological effects, mental illness, mental well-being, anxiety and depression.

\section{Inclusion criteria}

Systematic reviews were included if they provided a quantitative summary of the prevalence of anxiety and depression in HCWs in any healthcare setting and in any country due to COVID-19. Only systematic reviews published in English language were considered. Reviews undertaken on multiple populations were included if they provided separate data for HCWs. Literature reviews, case studies and reviews that provided an annotated bibliography of studies undertaken to assess the mental well-being of HCWs were excluded. In addition, reviews that included students and the general population were also excluded.

\section{Systematic review selection}

All identified citations were uploaded into EndNote V.X9 and duplicates removed. Three independent reviewers (RF, NS, HG) selected potentially relevant reviews for inclusion based on the titles and abstracts. Disagreements that arose between the reviewers at each stage of the study process were resolved through discussion, or with a fourth reviewer.

\section{Quality assessment}

The methodological quality of each review was rated independently by three independent authors (ST, LM, IA) and checked by a fourth author (RF) using the 11-point JBI Critical Appraisal Checklist for Systematic Reviews and Research Syntheses. ${ }^{18}$ This instrument assesses reviews for the following items (1) clear and explicit statement of the review question; (2) appropriate inclusion criteria; (3) comprehensive search strategy; (4) comprehensive literature search; (5) appropriate criteria used for appraising the studies; (6) independent critical appraisal by two or more reviewers; (7) methods implemented to minimise errors in data extraction; (8) appropriate methods used to combine study findings; (9) assessment of publication bias; (10) recommendations for policy and/or practice supported by the reported data and (11) appropriate specific directives for new research. The following quality thresholds were used: low quality $(0 \%-33 \%$ of criteria met), medium quality (34\%-66\% of criteria met) and high quality (67\% or more of criteria met).${ }^{19}$ All studies regardless of their methodological quality were included in the review. Details of the quality assessment are presented in table 1 .

\section{Data extraction}

For each included systematic review, the following data were extracted; year and country of publication, characteristics of the included studies, settings and populations, sample sizes of the studies that reported on anxiety and depression and the prevalence of anxiety and depression. Where sample sizes of the studies that reported on anxiety and depression were not available in the review, the primary studies were accessed and sample sizes calculated. Authors of studies were not contacted as data clarification was not required. Data extraction was undertaken independently by two authors (RF, NS), and checked by a third reviewer (HG).

\section{Data synthesis}

The degree of overlap in primary studies was assessed and calculated via the corrected covered area (CCA) index 


\begin{tabular}{|c|c|c|c|c|c|c|c|}
\hline $\begin{array}{l}\text { Krishnamoorthy et } \\
a l, 2020^{28}\end{array}$ & 18 Cross-sectional & China, Singapore & $38443 \mathrm{HCWs}$ & $\begin{array}{l}\text { Inception to } \\
\text { April } 2020\end{array}$ & NOS & $\begin{array}{l}\text { SDS, SAS, } \\
\text { PHQ-9, GAD- } \\
\text { 7, DASS-21, } \\
\text { HAMD, HAMA, } \\
\text { CES-D }\end{array}$ & 100 \\
\hline Luo et al, $2020^{30}$ & $\begin{array}{l}13 \text { Quantitative } \\
\text { studies }\end{array}$ & $\begin{array}{l}\text { China, Singapore, } \\
\text { South and Latin } \\
\text { America, Italy }\end{array}$ & $18224 \mathrm{HCWs}$ & $\begin{array}{l}1 \text { November to } \\
25 \text { May } 2020\end{array}$ & $\begin{array}{l}\text { McMaster } \\
\text { University } \\
\text { critical appraisal } \\
\text { tool }\end{array}$ & $\begin{array}{l}\text { BAI, DASS-21, } \\
\text { GAD-7, HADS, } \\
\text { HAMA, SAS, } \\
\text { STAI-S, HAMD }\end{array}$ & 72 \\
\hline Ren et al, $2020^{23}$ & 3 Cross-sectional & China & $5738 \mathrm{HCWs}$ & $\begin{array}{l}\text { December } \\
2019 \text { to April } \\
2020\end{array}$ & $\begin{array}{l}\text { Agency for } \\
\text { Healthcare } \\
\text { Research and } \\
\text { Quality }\end{array}$ & $\begin{array}{l}\text { SDS, SAS, } \\
\text { PHQ-9, GAD-7, } \\
\text { HAMD, HAMA }\end{array}$ & 100 \\
\hline $\begin{array}{l}\text { Santabárbara et al, } \\
2021^{24}\end{array}$ & 71 Cross-sectional & $\begin{array}{l}\text { China, Singapore, } \\
\text { Brazil, Cameroon, } \\
\text { Oman, Jordan, } \\
\text { India, Iran, Croatia, } \\
\text { Ecuador, Kosovo, } \\
\text { Germany, Libya, } \\
\text { Nepal, Pakistan, } \\
\text { Poland, Saudi } \\
\text { Arabia, South } \\
\text { America, Serbia, } \\
\text { South Korea, USA, } \\
\text { Turkey }\end{array}$ & 58565 HCWs & $\begin{array}{l}\text { December } \\
2019 \text { to August } \\
2020\end{array}$ & $\begin{array}{l}\text { JBI checklist } \\
\text { for prevalence } \\
\text { studies }\end{array}$ & $\begin{array}{l}\text { BAI, DASS-21, } \\
\text { GAD-7, HADS, } \\
\text { HAMA, SAS, } \\
\text { STAI-S }\end{array}$ & 90 \\
\hline
\end{tabular}

BAI, Beck Anxiety Inventory; BDI-2, Beck Depression Inventory-2; CES-D, Centre for Epidemiological Studies Depression Scale; DASS-21, Depression, Anxiety and Stress Scale; GAD-7, General Anxiety Disorder-7; HADS, Hospital Anxiety and Depression Scale; HAMA, Hamilton Anxiety Rating Scale; HAMD, Hamilton Depression Rating Scale; HCPs, healthcare professionals; HCWs, healthcare workers ; IES-R, Impact of Event Scale-Revised; NOS, Newcastle-Ottawa Scale; PHQ, Patient Health Questionnaire; SAS, Zung Self-Rating Anxiety Scale; SDS, Zung Self-Rating Depression Scale; STAI-S, State-Trait Anxiety Scale; STROBE, Strengthening the Reporting of Observational Studies in Epidemiology Checklist.

method. ${ }^{20} \mathrm{~A}$ CCA within the range $0 \%-5 \%$ indicates a slight overlap, $6 \%-10 \%$ indicates a moderate overlap, $11 \%-15 \%$ indicates a high overlap and $>15 \%$ indicates a very high amount of overlap. ${ }^{21}$ Results of the prevalence of anxiety and depression were calculated using the JBI SUMARI software. ${ }^{22}$ Data were inputted into the software and transformed using the Freeman-Tukey double arcsine transformation to calculate a summary statistic for each 
review. ${ }^{22}$ Pooled estimates for the systematic reviews were not calculated due to overlap of primary studies in the 10 reviews. Separate analysis was undertaken where possible for physicians and nurses. The systematic review characteristics are presented in a tabular form and descriptively. Results of the prevalence of anxiety and depression are presented as \% with 95\% CIs and are graphically presented in forest plots.

\section{Patient and public involvement}

Patients and the public were not involved in the design and conduct of this review.

\section{RESULTS}

\section{Study characteristics}

A total of 236 citations were identified through the electronic search. Following removal of duplicates, 97 records underwent screening of the title and abstracts. Eighty-five papers were excluded as they did not meet the inclusion criteria. The full texts of 12 papers were retrieved for further examination with 10 systematic reviews meeting the inclusion criteria and therefore included in this umbrella review. Two systematic reviews were excluded as they provided aggregate data for general population and health professionals (figure 1 ).

The 10 included systematic reviews included a total of 100 primary studies undertaken in 35 countries. The number of studies included in individual reviews ranged from $3^{23}$ to $71 .^{24}$ The sample sizes of HCWs in the reviews ranged from $5738^{23}$ to $58565 .{ }^{24}$ All reviews focused on HCWs in the hospital setting. HCWs included physicians, nurses, allied health, administrative and ancillary staff. Two reviews ${ }^{23}{ }^{25}$ were undertaken only on healthcare professionals and the remaining reviews included all HCWs. However, two reviews ${ }^{24}{ }^{26}$ did report data separately for physicians, nurses and other HCWs. Various validated instruments were used to measure anxiety and depression (table 1).

\section{Study overlap}

Twenty-eight of the 100 primary studies appeared in at least two reviews. Overall, the CCA demonstrated a
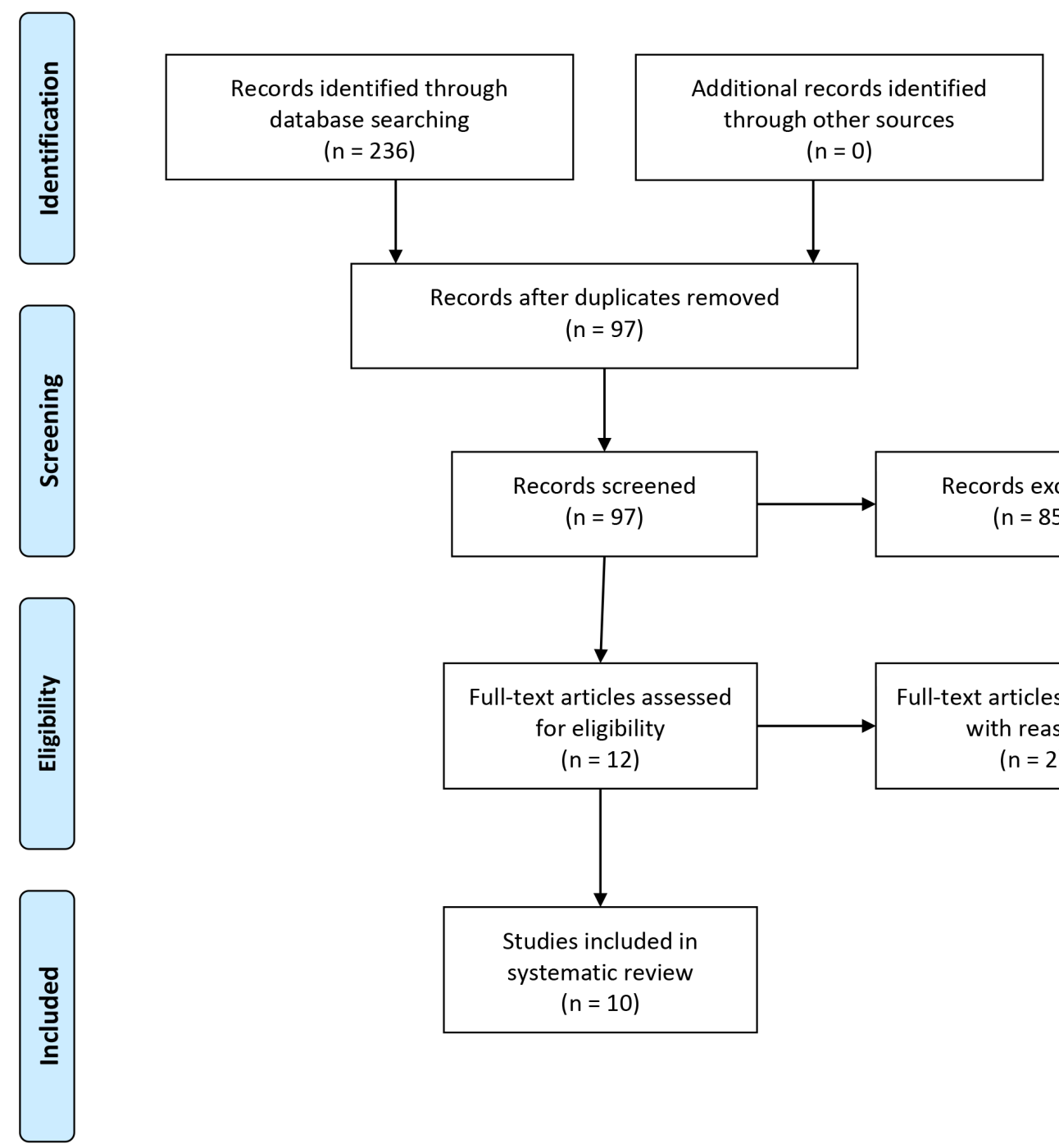

Figure 1 PRISMA 2020 flow diagram. PRISMA, Preferred Reporting Items for Systematic Reviews and Meta-Analyses. 


\section{Pappa et al., 2020 \\ Luo et al., 2020 \\ Salari et al., 2020 \\ da Silva and Neto, 2020 \\ Dutta et al., 2020 \\ Santabárbara et al., 2021 \\ Killikelly et al., 2021 \\ de Pablo et al., 2020}

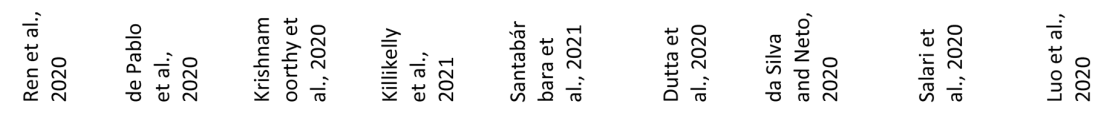

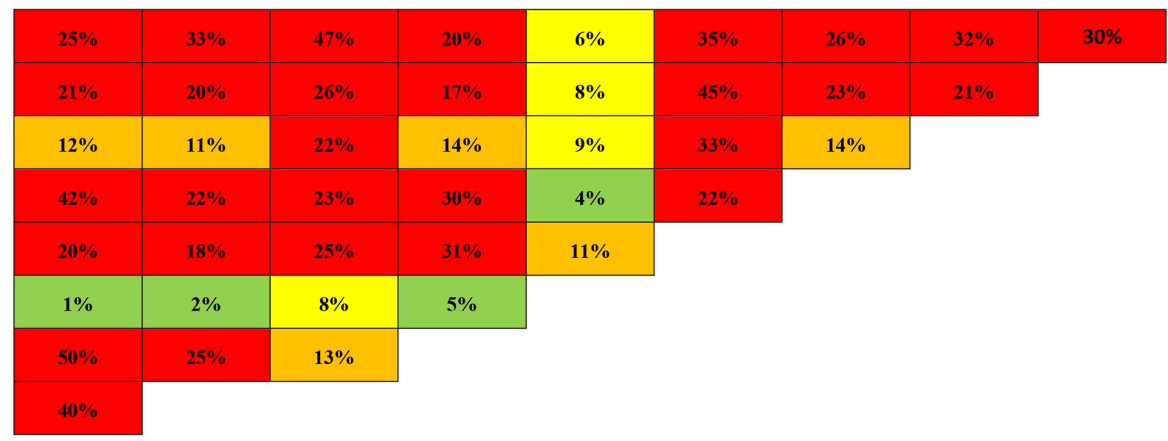

Interpretation

0-5\%: slight overlap $\quad 11-15 \%$ : high overlap

6-10\%: moderate overlap $\quad>15 \%$ : very high overlap

Figure 2 Study overlap.

moderate degree of overlap of $8.4 \%$ between the primary studies. The degree of overlap ranged from $1 \%$ to $50 \%$. Figure 2 presents the degree of overlap between the studies.

\section{Quality appraisal of the included reviews}

The critical appraisal instruments used in the reviews were the Newcastle-Ottawa Scale ${ }^{27-29}$ McMaster University tool, ${ }^{30}$ Agency for Healthcare Research and Quality, ${ }^{23}$ Strengthening the Reporting of Observational Studies in Epidemiology Checklist, ${ }^{26}$ JBI for prevalence studies ${ }^{24}$ and assessment tool used for mental health and disease outbreaks, ${ }^{31}$ mixed methods appraisal tool. ${ }^{25}$ Four reviews $^{23} 252628$ reported on all 11 criteria for quality assessment. Publication bias was not reported in five studies $^{25} 27$ 29-31 (table 1). Reporting publication bias is not valid for proportional meta-analysis as they are noncomparative hence there are no negative results or study characteristics that may have biased publications. ${ }^{32}$

\section{Prevalence of anxiety}

Ten included reviews summarised the prevalence of anxiety in HCWs. The pooled prevalence of anxiety ranged from $22.2 \%(95 \% \text { CI } 21.3 \% \text { to } 23.1 \%)^{25}$ to $33.0 \%$
$(95 \% \text { CI } 31.9 \% \text { to } 34.1 \%)^{33}$ (figure 3 ). Due to an overlap of studies included in the reviews, a pooled prevalence of anxiety was not calculated.

Subgroup analysis demonstrated that the prevalence of anxiety ranged from $22.2 \%(95 \% \text { CI } 13 \% \text { to } 23.1 \%)^{25}$ to $27 \%(95 \% \text { CI } 25.9 \% \text { to } 28.2 \%)^{23}$ among healthcare professionals (figure 4 ). The prevalence of anxiety specifically among nurses ( $\mathrm{n}=14938$ nurses; two reviews) was reported to be $22.8 \%$ (95\% CI $21.9 \%$ to $23.7 \%)^{26}$ and $27 \%$ $(95 \%$ CI $26.0 \%$ to $28.1 \%) .{ }^{24}$ The prevalence of anxiety specifically among physicians ( $n=5820$ physicians; two reviews) was somewhat lower than nurses and reported to be between $17 \%$ (95\% CI $16.0 \%$ to $18.0 \%)^{24}$ and $19.8 \%$ $(95 \%$ CI $16.8 \%$ to $22.9 \%) .^{26}$

\section{Prevalence of depression}

While all 10 included reviews reported on depression, only nine reviews summarised depression prevalence. The prevalence of depression ranged from $17.9 \%$ $(95 \% \text { CI } 17.1 \% \text { to } 18.8 \%)^{25}$ to $36 \%$ (95\% CI $34.9 \%$ to $37.1 \%)^{33}$ (figure 5). Due to overlap of studies included in the reviews, a pooled prevalence of depression was not calculated.

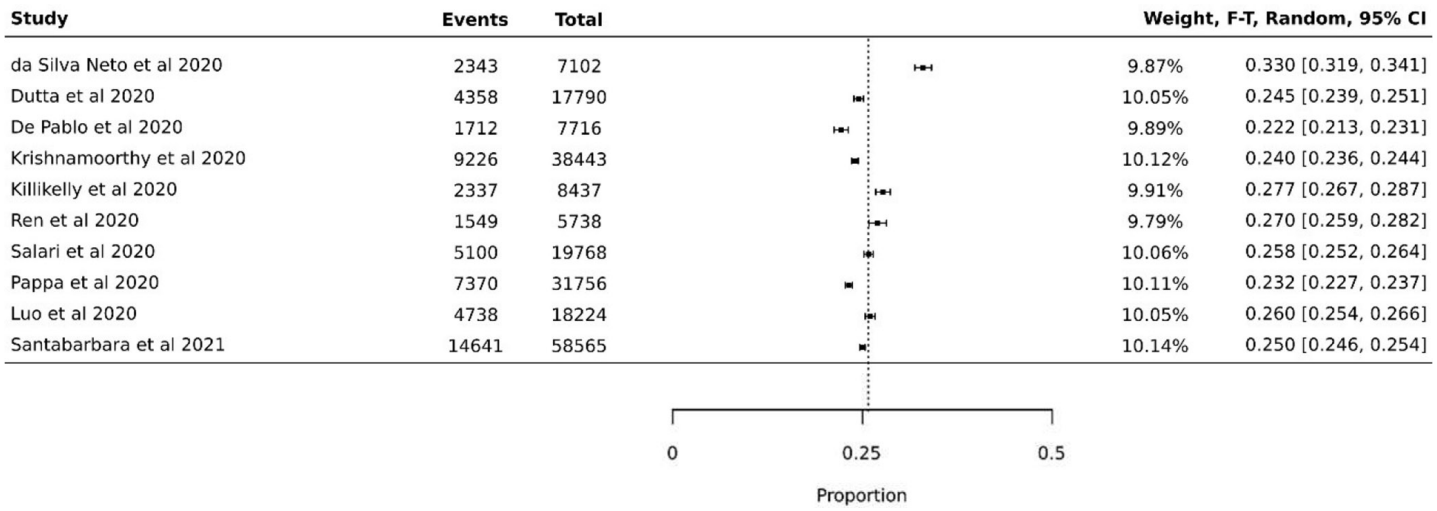

Figure 3 Prevalence of anxiety among all healthcare workers. 


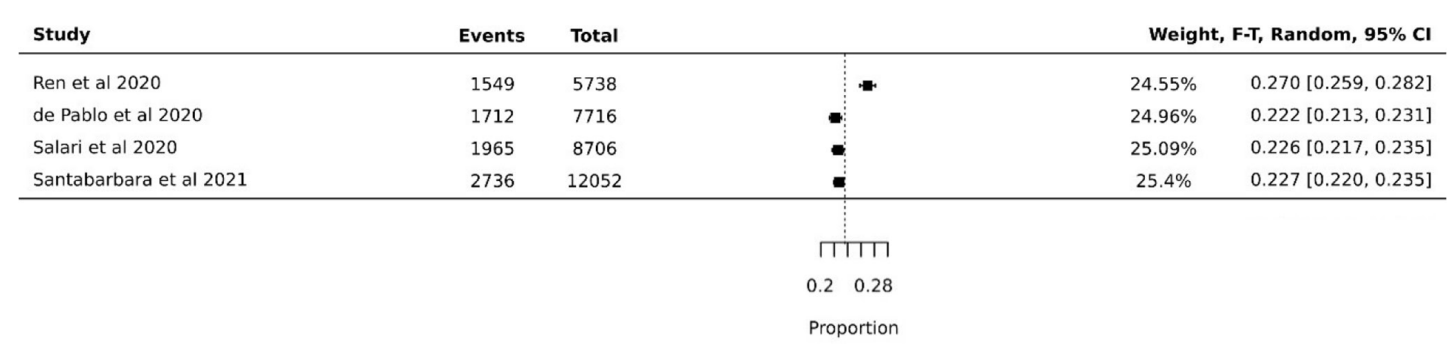

Figure 4 Prevalence of anxiety among all healthcare professionals (doctors, nurses, allied health staff).

A subgroup analysis demonstrated that the prevalence of depression ranged from $17.9 \%$ (95\% CI $17.1 \%$ to $18.8 \%)^{25}$ to $28.9 \%(95 \% \text { CI } 28.0 \% \text { to } 29.9 \%)^{26}$ among healthcare professionals (figure 6). While the prevalence of depression specifically among nurses ( $\mathrm{n}=8063$ nurses; one review) was reported to be $28 \%$ (95\% CI $27 \%$ to $29.0 \%),{ }^{26}$ the prevalence of depression specifically among physicians ( $\mathrm{n}=643$ physicians; one review) was reported to be $40.4 \%$ (95\% CI $36.7 \%$ to $44.3 \%)^{26}$

\section{DISCUSSION}

The COVID-19 pandemic placed HCWs at a substantially increased risk for anxiety and depression due to the enormity of the situation, particularly due to risk of infection, limited resources and stress related to the emerging disease ${ }^{29}$ This umbrella review provides a comprehensive synthesis of the prevalence rates of anxiety and depression among health workers globally during the COVID-19 pandemic. Ten existing systematic reviews met the inclusion criteria for this umbrella review, however due to the overlap of studies across the systematic reviews, a pooled prevalence for either anxiety or depression was not calculated.

This umbrella review demonstrates that among HCWs the prevalence rates for anxiety ranged from $22.2 \%$ to $33 \%$ and for depression ranged from $17.9 \%$ to $36 \%$. This finding may be explained by a number of reasons. First, a key challenge in the included studies is that various instruments were used to measure anxiety and depression. The majority of instruments used were developed based on what Western people consider to be symptoms of anxiety and depression. Given that most reviews included large numbers of studies undertaken in countries such as China and Singapore, the instruments used may not have been appropriate for the non-western context. Indeed, a study examining Chinese mental well-being using western developed instruments found that Chinese participants were more likely to score the mid points ${ }^{34}$ when compared with the North American population, suggesting that the instruments may not be relevant for this population group. Hence, using instruments validated for the specific cultural group can enhance crosscultural relevance. ${ }^{35}$

It should be noted that various instruments were used to measure anxiety and depression in the studies included in the individual systematic review. Some of the instruments used to measure anxiety have been reported to have poor ability to discriminate between somatic anxiety and somatic side effects leading to overestimation of anxiety. ${ }^{36}$ It was beyond the scope of this review to investigate the prevalence of anxiety and depression based on the type of instruments used as the aim was to explore the prevalence of anxiety and depression in HCWs.

Variability in the prevalence rates of anxiety and depression could also be related to the lack of universality in the experience and expression of anxiety and depression, particularly across diverse settings. For some HCWs, admitting that they are dealing or experiencing symptoms of anxiety or depression is often still considered a taboo subject ${ }^{37}$ with a culture of 'not complaining' or fear of being labelled as inferior. ${ }^{38}$ Hence, cultural norms can also provide an explanation for the variance in the prevalence rates across the reviews, with some HCWs saying that they would 'feel weak', if they expressed symptoms of anxiety and depression. ${ }^{37}$

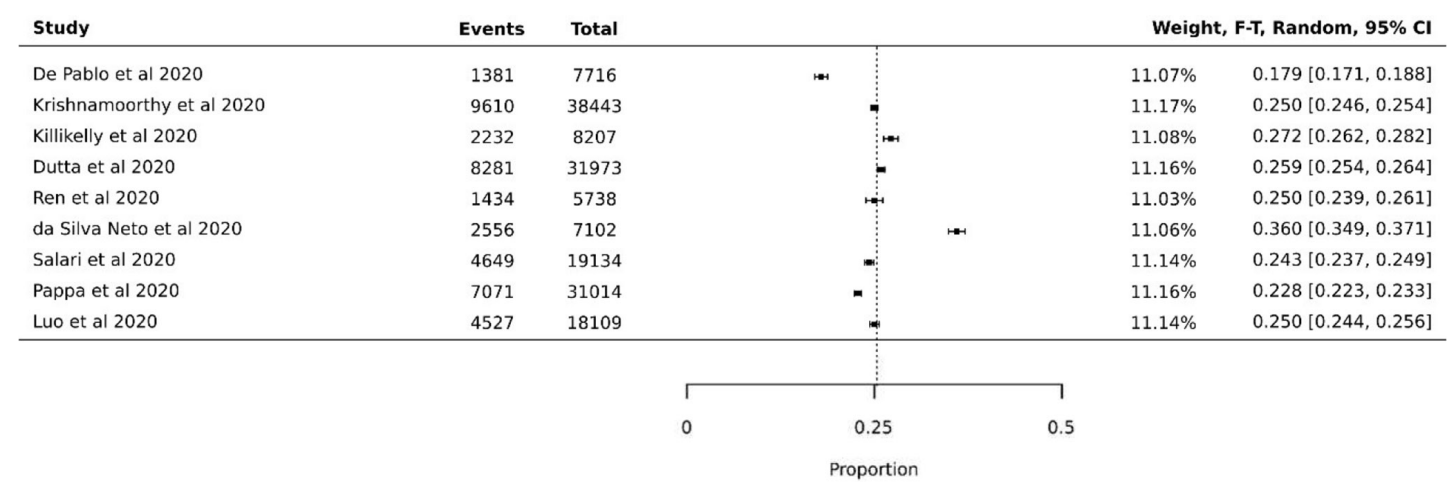

Figure 5 Prevalence of depression among all healthcare workers. 


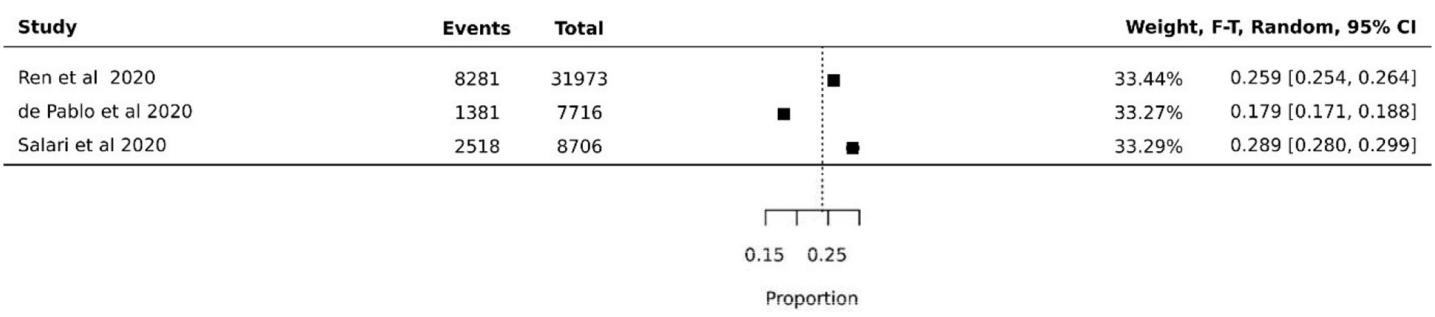

Figure 6 Prevalence of depression among all healthcare professionals (doctors, nurses, allied health staff).

The high level of anxiety and depression could be associated with the differing mortality and infection rates between the countries where the primary studies were conducted. Perceptions of anxiety and depression among HCWs could be attributed to their working conditions, ${ }^{39}$ previous exposure to epidemics and pandemics, adequacy of their PPE and perceived support. ${ }^{12}$ The primary studies included in the reviews were undertaken in countries that had high rates of COVID-19 infections and mortality which could also explain the high prevalence of anxiety and depression. It would be interesting to see if HCWs in countries such as with low COVID-19 infections had similar rates of anxiety and depression.

Surprisingly, the results of this review highlight that physicians had a high prevalence of symptoms of depression. Similarly, a study conducted in Pakistan found that the prevalence of depression among frontline physicians during the COVID-19 pandemic was high at 43\%, with doctors working in emergency department and those of a younger age demonstrating higher rates of depressive symptoms. ${ }^{40}$ Depressive symptoms among physicians may have been caused by higher work related stress due to the health system being placed under significant pressure and heightend fears of risk to their own health. Evidence from the literature from previous epidemics, such as SARS, indicates that depression is caused as a response to the life-threatening situation. ${ }^{41}{ }^{42}$ However, the results of this review on depression among physicians must be interpreted with caution as the results are only based on one systematic review.

Interestingly, the results of this review indicate that while physicians experienced depression, nurses were found to experience more anxiety. Nurses may have experienced higher anxiety symptoms due them having constant direct contact with patients infected with COVID-19 which could be associated with heightened concerns over being infected, especially compared with physicians. This is similar to nurses' experiences during the SARS epidemic, whereby anxiousness was associated with fear of infection and uncertainties about the emerging virus. ${ }^{434}$

The clinical significance of the results from this umbrella review is important to consider, as HCWs who experience symptoms anxiety or depression will need to be supported. These results are important as anxiety and depression symptoms highlight a need for urgent support for HCWs to manage these stressors and reduce their ongoing impact. Indeed, HCWs may need to be equipped with specific coping strategies to assist them, they may need additional support such as counselling and time away from the workplace. As evidenced in the literature, without such support, poor mental health in HCWs may result in the quality of care that can be provided and could lead to burnout, ${ }^{45}$ including longer term consequences and a decreased workforce.

\section{Strengths and limitations}

The strengths of this umbrella review are the inclusion of 10 systematic reviews involving 100 primary studies and 169157 HCWs from 35 countries. Another strength of this umbrella review is the rigour in which it was conducted. First, all steps of the review process were conducted by two people and checked by a third author. Second, the degree of overlap in primary studies was assessed and calculated using the CCA index method. Third, the sample sizes in this review are specific to anxiety and depression and not other outcomes reported in the reviews. Despite the rigour in which the umbrella review was conducted, some limitations should be considered when interpreting the results. First, the heterogeneity of the primary studies included in some of the systematic reviews and significant study overlap between reviews, meant pooled data were not able to be calculated. Second, anxiety and depression were reported in multiple primary studies and most of these were predominantly from China and conducted in hospital settings, as a result, psychological disturbances between different settings could not be explored in depth in this review. In addition, anxiety and depression could vary between professional and non-professional HCWs. We therefore recommend that future research should focus on the differences between contexts and between various HCWs. Finally, in this umbrella review, we only included systematic reviews published in the English language which is an additional limitation.

\section{CONCLUSION}

In conclusion, although anxiety and depression have been explored in relation to HCWs and COVID-19, there is wide variation evident for anxiety and depression among this population. This may be due to the diversity of experiences and impact of COVID-19 internationally. It may also be due to cultural differences that are not factored in most tools used to measure anxiety and depression, with a Western perspective inadvertently applied in many tools. With so many factors influencing HCWs anxiety and depression, it is challenging to establish commonality 
in risk factors. On the basis of the results of this review, an association between HCWs experiencing anxiety and depression during the COVID-19 pandemic is clear. It is evident that anxiety and depression are increased due to pandemic stressors, however, further studies are needed to draw firmer conclusions as to what specifically triggers these responses. In addition, more studies need to be conducted to develop and implement interventions that address well-being and mental health among HCWs and foster post-traumatic growth. It is clear from this review that strategies to reduce the incidence of anxiety and depression are required, together with support for staff to proactively approach future pandemics.

\section{Author affiliations}

${ }^{1}$ School of Nursing, Faculty of Science, Medicine and Health, University of Wollongong, Wollongong, New South Wales, Australia

${ }^{2}$ St George Hospital, South Eastern Sydney Local Health District, Kogarah, New South Wales, Australia

${ }^{3}$ Centre for Evidence based Initiatives in Health Care: A JBI Centre of Excellence, University of Wollongong, Wollongong, New South Wales, Australia

${ }^{4}$ Illawarra Health and Medical Research Institute (IHMRI), Wollongong, New South Wales, Australia

\section{Twitter Elizabeth J Halcomb @LizHalcomb}

Contributors RF had the idea for the review. RF and HG were involved in designing the study. NS, $\mathrm{HG}$ and RF were involved in searching the database and screened citations for inclusion. HG, IA and ST were involved in risk of bias analysis. HG, IA, RF, NS, ST were involved in extracting data. RF, LM, HG, EJH, RM were involved in data interpretation. RF, HG and NS analysed the data. All authors were in involved in drafting the manuscript. RF is the study guarantor. All authors reviewed the final manuscript and agreed to be accountable for all aspects of the work and approved the final manuscript for submission. The corresponding author attests that all listed authors meet authorship criteria and that no others meeting the criteria have been omitted.

Funding The authors have not declared a specific grant for this research from any funding agency in the public, commercial or not-for-profit sectors.

Competing interests None declared.

Patient and public involvement Patients and/or the public were not involved in the design, or conduct, or reporting, or dissemination plans of this research.

\section{Patient consent for publication Not required}

Provenance and peer review Not commissioned; externally peer reviewed.

Data availability statement Data sharing not applicable as no datasets generated and/or analysed for this study. All data relevant to the study are included in the paper. "No additional data available"

Open access This is an open access article distributed in accordance with the Creative Commons Attribution Non Commercial (CC BY-NC 4.0) license, which permits others to distribute, remix, adapt, build upon this work non-commercially, and license their derivative works on different terms, provided the original work is properly cited, appropriate credit is given, any changes made indicated, and the use is non-commercial. See: http://creativecommons.org/licenses/by-nc/4.0/.

\section{ORCID iDs}

Ritin Fernandez http://orcid.org/0000-0002-6143-7703

Elizabeth J Halcomb http://orcid.org/0000-0001-8099-986X

\section{REFERENCES}

1 Zheng L, Wang X, Zhou C, et al. Analysis of the infection status of healthcare workers in Wuhan during the COVID-19 outbreak: a cross-sectional study. Clin Infect Dis 2020;71:2109-13.

2 Gholami M, Fawad I, Shadan S, et al. COVID-19 and healthcare workers: a systematic review and meta-analysis. Int J Infect Dis 2021;104:335-46.
3 Batra K, Singh TP, Sharma M, et al. Investigating the psychological impact of COVID-19 among healthcare workers: a meta-analysis. Int $J$ Environ Res Public Health 2020;17:9096.

4 Shen M, Xu H, Fu J, et al. Investigation of anxiety levels of 1637 healthcare workers during the epidemic of COVID-19. PLoS One 2020;15:e0243890.

5 Walton M, Murray E, Christian MD. Mental health care for medical staff and affiliated healthcare workers during the COVID-19 pandemic. Eur Heart J Acute Cardiovasc Care 2020;9:241-7.

6 Zheng R, Zhou Y, Fu Y, et al. Prevalence and associated factors of depression and anxiety among nurses during the outbreak of COVID-19 in China: a cross-sectional study. Int J Nurs Stud 2021;114:103809.

7 Bandyopadhyay S, Baticulon RE, Kadhum M, et al. Infection and mortality of healthcare workers worldwide from COVID-19: a systematic review. BMJ Glob Health 2020;5:e003097.

8 Halcomb E, Mclnnes S, Williams A, et al. The experiences of primary healthcare nurses during the COVID-19 pandemic in Australia. J Nurs Scholarsh 2020;52:553-63.

9 Li J, Long X, Zhang Q et al. Mild manifestations of COVID-19 in healthcare workers. PLoS Negl Trop Dis 2020;14:e0008950.

10 Shaukat N, Ali DM, Razzak J. Physical and mental health impacts of COVID-19 on healthcare workers: a scoping review. Int J Emerg Med 2020;13:1-8.

11 Carmassi C, Foghi C, Dell'Oste V, et al. PTSD symptoms in healthcare workers facing the three coronavirus outbreaks: what can we expect after the COVID-19 pandemic. Psychiatry Res 2020;292:113312.

12 Fernandez R, Lord H, Halcomb E, et al. Implications for COVID-19: a systematic review of nurses' experiences of working in acute care hospital settings during a respiratory pandemic. Int J Nurs Stud 2020;111:103637.

13 Spoorthy MS, Pratapa SK, Mahant S. Mental health problems faced by healthcare workers due to the COVID-19 pandemic-A review. Asian J Psychiatr 2020;51:102119.

14 Xiao J, Fang M, Chen Q, et al. SARS, MERS and COVID-19 among healthcare workers: a narrative review. J Infect Public Health 2020;13:843-8

15 Sahebi A, Nejati-Zarnaqi B, Moayedi S, et al. The prevalence of anxiety and depression among healthcare workers during the COVID-19 pandemic: an umbrella review of meta-analyses. Prog Neuropsychopharmacol Biol Psychiatry 2021;107:110247.

16 Page MJ, Moher D, Bossuyt PM, et al. PRISMA 2020 explanation and elaboration: updated guidance and exemplars for reporting systematic reviews. BMJ 2021;372:n160.

17 Aromataris EF, Godfrey R;, Holly C; , et al. Chapter 10: umbrella reviews, 2020

18 Aromataris E, Fernandez R, Godfrey CM, et al. Summarizing systematic reviews: methodological development, conduct and reporting of an umbrella review approach. Int J Evid Based Healthc 2015:13:132-40.

19 Schultz TJ, Roupas P, Wiechula R, et al. Nutritional interventions for optimizing healthy body composition in older adults in the community: an umbrella review of systematic reviews. JBI Database System Rev Implement Rep 2016;14:257-308.

20 Hennessy EA, Johnson BT. Examining overlap of included studies in meta-reviews: guidance for using the corrected covered area index. Res Synth Methods 2020;11:134-45.

21 Pieper D, Antoine S-L, Mathes T, et al. Systematic review finds overlapping reviews were not mentioned in every other overview. $J$ Clin Epidemiol 2014;67:368-75.

22 Munn Z, Aromataris E, Tufanaru C, et al. The development of software to support multiple systematic review types: the Joanna Briggs Institute system for the unified management, assessment and review of information (JBI SUMARI). Int J Evid Based Healthc 2019;17:36-43.

23 Ren X, Huang W, Pan $\mathrm{H}$, et al. Mental health during the Covid-19 outbreak in China: a meta-analysis. Psychiatr Q 2020;91:1033-45.

24 Santabárbara J, Bueno-Notivol J, Lipnicki DM, et al. Prevalence of anxiety in health care professionals during the COVID-19 pandemic: a rapid systematic review (on published articles in MEDLINE) with meta-analysis. Prog Neuropsychopharmacol Biol Psychiatry 2021;107:110244

25 Salazar de Pablo G, Vaquerizo-Serrano J, Catalan A, et al. Impact of coronavirus syndromes on physical and mental health of health care workers: systematic review and meta-analysis. J Affect Disord 2020;275:48-57.

26 Salari N, Khazaie H, Hosseinian-Far A, et al. The prevalence of stress, anxiety and depression within front-line healthcare workers caring for COVID-19 patients: a systematic review and metaregression. Hum Resour Health 2020;18:1-14. 
27 Dutta A, Sharma A, Torres-Castro R, et al. Mental health outcomes among healthcare workers dealing with COVID-19/ SARSCoV-2 pandemic: a systematic review and meta-analysis. SSRN 2020:3633225

28 Krishnamoorthy Y, Nagarajan R, Saya GK, et al. Prevalence of psychological morbidities among general population, healthcare workers and COVID-19 patients amidst the COVID-19 pandemic: a systematic review and meta-analysis. Psychiatry Res 2020;293:113382.

29 Pappa S, Ntella V, Giannakas T, et al. Prevalence of depression, anxiety, and insomnia among healthcare workers during the COVID-19 pandemic: a systematic review and meta-analysis. Brain Behav Immun 2020;88:901-7.

30 Luo M, Guo L, Yu M, et al. The psychological and mental impact of coronavirus disease 2019 (COVID-19) on medical staff and general public - A systematic review and meta-analysis. Psychiatry Res 2020;291:113190.

31 Killikelly C, Lenferink LIM, Xie H, et al. Rapid systematic review of psychological symptoms in health care workers COVID-19. J Loss Trauma 2021;26:638-55.

32 Maulik PK, Mascarenhas MN, Mathers CD, et al. Prevalence of intellectual disability: a meta-analysis of population-based studies. Res Dev Disabil 2011;32:419-36.

33 da Silva FCT, Neto MLR. Psychological effects caused by the COVID-19 pandemic in health professionals: a systematic review with meta-analysis. Prog Neuropsychopharmacol Biol Psychiatry 2021;104:110062.

34 Zhang J, Norvilitis JM. Measuring Chinese psychological wellbeing with Western developed instruments. J Pers Assess 2002;79:492-511.

35 Borsa JC, Damasio BF, Bandeira DR. Cross-Cultural adaptation and validation of psychological instruments: some considerations. Paidéia 2012;22:423-32.
36 Leichsenring F, Hiller W, Weissberg M, et al. Cognitive-Behavioral therapy and psychodynamic psychotherapy: techniques, efficacy, and indications. Am J Psychother 2006;60:233-59.

37 Stankovic S. 'I cried every day at work': mental health among doctors is still taboo. The Guardian, 2017. Available: https://www.theguardian. com/commentisfree/2017/jul/04/mental-health-among-doctors-still-ataboo-in-a-high-performing-profession [Accessed 15 Mar 2021]

38 Vaughn N. Depression and anxiety in nurses, 2020. Available: https:// www.relias.com/blog/depression-anxiety-in-nurses [Accessed 15 Mar 2021].

39 Cheng W-J, Cheng Y. Minor mental disorders in Taiwanese healthcare workers and the associations with psychosocial work conditions. J Formos Med Assoc 2017;116:300-5.

40 Amin F, Sharif S, Saeed R, et al. COVID-19 pandemic- knowledge, perception, anxiety and depression among frontline doctors of Pakistan. BMC Psychiatry 2020;20:1-9.

41 Sim K, Chong PN, Chan YH, et al. Severe acute respiratory syndrome-related psychiatric and posttraumatic morbidities and coping responses in medical staff within a primary health care setting in Singapore. J Clin Psychiatry 2004;65:1120-7.

42 Styra R, Hawryluck L, Robinson S, et al. Impact on health care workers employed in high-risk areas during the Toronto SARS outbreak. J Psychosom Res 2008;64:177-83.

43 Chan SSC, Leung GM, Tiwari AFY, et al. The impact of work-related risk on nurses during the SARS outbreak in Hong Kong. Fam Community Health 2005;28:274-87.

44 Lau PY, Chan CWH. SARS (severe acute respiratory syndrome): reflective practice of a nurse manager. $J$ Clin Nurs 2005;14:28-34.

$45 \mathrm{Ma} \mathrm{H}$, Qiao H, Qu H, et al. Role stress, social support and occupational burnout among physicians in China: a path analysis approach. Int Health 2020;12:157-63. 